\title{
Quality issues for echocardiography in the community
}

\author{
S Saltissi, J Chambers
}

Community echocardiography refers to cardiac ultrasound imaging which is requested by, reported to, and acted upon by general practitioners. Mobile laboratories can conveniently take echo technology to the patient in the community and may occasionally be appropriate in a large general practice. More usually, however, this represents an inefficient use of staff time and resources, as well as exposing fragile and expensive equipment to substantial stresses during movement. Consequently, community echo has become essentially synonymous with open access echocardiography (OAE) using hospital based equipment.

In the last few years, many OAE services have been initiated. In a survey of 100 hospitals in $1996,{ }^{1} 30$ were already providing the service and 21 of these had commenced within the last 12 months. A further 11 hospitals were actively planning this facility.

Undoubtedly, in the near future many more hospitals will come under pressure from within and without (general practitioner primary care groups, purchasers, patients) to follow suit. For some, an OAE service would represent a clinically sound and cost effective initiative, while in others the local community might be better (and more cheaply) served by an alternative arrangement such as a hospital based, rapid access clinic focused on a particular type of problem-for example, heart failure or murmur.

This paper aims to review OAE in a way that will help the reader to decide whether it is an appropriate investment for a particular locality and, if so, to outline some of its strengths and weaknesses, allowing a more effective service to be established.

\section{How open is open access echo?}

The term OAE implies that the service should be equally available to all general practitioners and for all appropriate indications. However, in the aforementioned study ${ }^{1}$ access to the services was restricted solely to general practitioner fundholders in $27 \%$, to particular general practitioner practices only in $10 \%$, and to research trial participants only in $3 \%$. In others, the restriction related to the indication, usually being limited to patients with heart failure, the condition which has been the main stimulus for the introduction of OAE.

In Liverpool, a city wide OAE service was started in April 1998 and limited solely to patients with known or suspected heart failure. This was in response to health authority requirements and was timed to accompany comprehensive heart failure guidelines issued to local general practitioners.
We believe that in some cases there may be sound arguments for restricting OAE to indications such as heart failure, which are most likely to produce a cost effective health care benefit for the community, and for which funding may therefore be available. Indeed, this type of resource prioritisation would seem to be a prime role of primary care groups or others with the responsibility of purchasing health care. Restriction on other bases may, however, lead to unacceptable inequity.

\section{Differences between hospital and community echo services}

It is a common error to regard OAE as an extension of normal hospital work. There are a number of fundamental differences.

Hospital patients are prescreened, resulting in a much higher likelihood of cardiac pathology, and hence a primary requirement for accurate diagnosis and assessment of abnormalities. On the other hand, patients from the community are derived from a population with a much lower pretest probability of disease and are more often referred to exclude disease. Both the echo procedure and its interpretation will differ between the two situations. In particular, in a patient likely to have disease, the test must be inclusive and comprehensive, while in a patient likely to be normal, there is a danger of creating confusion by inappropriately reporting irrelevant, minor, or equivocal departures from strict normality. Thus, while high quality imaging is essential for both, an OAE study might not routinely include assessment of left ventricular (LV) diastolic function, or assessment of the right ventricle.

Hospital echocardiograms are easily checked or reviewed by the referring specialist clinician who can integrate the report with clinical findings and the results of other investigations. In contrast, OAE must stand or fall on the report alone with, at best, a short clinical comment from an uninvolved clinician.

Problems can also arise in applying to $\mathrm{OAE}$ normal ranges and interpretations of echo findings derived from hospital studies. The demography and, in particular, the age ranges of the populations may be different. In the elderly as a group, minor aortic valve thickening is common, minor mitral regurgitation normal, tricuspid regurgitation almost universal, and LV function (particularly diastolic) difficult to assess or interpret.

In contrast, however, initial concerns that general practitioners might be indiscriminate in their referral seems no more true of OAE than of open access endoscopy. ${ }^{2}$ In one study, ${ }^{3}$ only 32 of 259 general practitioner referrals $(12 \%)$ were considered inappropriate. 
Potential advantages of open access echo The major potential benefit of OAE lies in the detection of previously undiagnosed disease for which effective treatment exists. Heart failure has provided the major impetus to OAE development because it is common (affecting $8-10 \%$ of the elderly ${ }^{4}$ ), and carries a poor prognosis $\left(40-50 \%\right.$ mortality at five years $\left.{ }^{5}\right)$ which can be greatly improved (by approximately $23 \%^{6}$ ) using angiotensin converting enzyme (ACE) inhibitors. Furthermore, this treatment may well be very cost effective, bearing in mind the enormous financial burden of heart failure on the National Health Service ( $£ 360$ million per year, $60 \%$ on hospital care ${ }^{8}$ ). Patients suitable for ACE inhibitor treatment, however, have mainly been identified on the basis of reduced LV systolic function (ejection fraction $0.35-0.4$ or less). Widespread use of this treatment therefore requires rapid access to an affordable means of non-invasive LV assessment, a purpose for which OAE seems ideally suited.

Surprisingly though, Francis et al found that only 14 of 99 patients undergoing OAE for suspected heart failure actually required ACE inhibitors. ${ }^{3}$ The main initial benefit was the opportunity to stop diuretic therapy in patients with normal systolic function. However, a proportion of these patients may subsequently relapse, ${ }^{9}$ underlining that echocardiography should not be the only determinant of clinical decision making.

Another possible benefit is the avoidance of specialist cardiological referral. This can save money if an echocardiogram is cheaper than a hospital consultation. It can also save time if the waiting list for echocardiography is shorter than for an outpatient appointment. In one pilot study, 97 of 222 investigations were considered to have saved a clinic referral, ${ }^{10}$ although from another perspective avoiding a clinic visit may be seen as depriving the patient of a specialist opinion. The availability of open access systems has also been felt to encourage a strong community based feeling which might hinder appropriate referral to hospital. ${ }^{11} \mathrm{~Pa}$ tients with complex symptomatic cardiac disease will usually need referral to a cardiologist who is more likely to achieve a better outcome with a minimum of investigation than is a general physician or general practitioner. ${ }^{12}$ With regard to general practitioner care, patients will benefit most from those who know the limits of their abilities and can judge when referral to a cardiologist is necessary. OAE can be used to aid this judgment.

\section{Appropriate indications}

As yet, there is no consensus as to appropriate indications for OAE. However, there are a number of different problem areas which could potentially benefit (table 1 ). These constitute a spectrum varying from simple questions that can be answered by a technician alone to complex problems in which the echo cannot be dissociated from clinical assessment. In practice, however, most referrals are for suspected heart failure or for heart murmurs. ${ }^{10} 13$
Table 1 Indications for open access echocardiography

Generally agreed

Suspected heart failure or left ventricular dysfunction

Assessment of asymptomatic heart murmurs

Controversial

Assessment of cardiac end organ damage (left

ventricular hypertrophy) in hypertension

Atrial fibrillation (?cause, ?need for anticoagulation)

Screening for hypertrophic cardiomyopathy

Screening for Marfan's syndrome

The value of OAE is greatest when a single focused question can be asked, answered by a technician, and lead to a change in management by the general practitioner. Such questions might include: is there LV hypertrophy present? (requiring drug treatment of hypertension); is there evidence of hypertrophic cardiomyopathy? (requiring referral, treatment, or reassurance); or is there valve thickening present? (requiring antibiotic prophylaxis).

At the other extreme is the patient with breathlessness. An OAE will be able to identify LV systolic dysfunction and hence the need for ACE inhibitor treatment; however, a normal result may be misleading (is there diastolic dysfunction or myocardial ischaemia?) and needs to be interpreted in the context of a clinical assessment and other tests-for example, ECG, chest radiograph, pulmonary function tests. Initial referral to a cardiologist via a rapid access clinic rather than for an OAE might serve such a patient better.

\section{Reporting the open access}

\section{echocardiogram}

The report and its interpretation are central to the success of OAE. The principal report should be generated by an experienced echocardiography technician (ideally with BSE accreditation) who subserves several functions, including quality control, filtering out of artefacts and "red herrings", and emphasising relevant findings.

This process requires extensive knowledge of cardiology and of the strengths and limitations of the technique itself, which would understandably be well beyond a general practitioner and often beyond a general physician. Postal surveys have shown that general practitioners require results that are simple to interpret and that they are aware of a need for more instruction about echo, ${ }^{14}$ although only half of the current OAE services supply educational material. ${ }^{1}$

One solution is for reports to be vetted by a cardiologist who can add a clinical comment; $57 \%$ of surveyed OAE services have adopted this approach. ${ }^{1}$ This area, however, is controversial and open to abuse. There are real dangers in basing management decisions solely on an echo study totally divorced from knowledge of essential clinical data and other test results. ${ }^{915}{ }^{16}$ Arguably, a rapid access one stop cardiological clinic would be better where a straightforward technical report would not suffice.

\section{Organisation and funding}

OAE already represents a significant load on strained resources. For example, at Guy's and 
St Thomas's Hospitals, 535 of 10800 studies (5\%) performed during 1996 were open access, an increase by $40 \%$ on the previous year. The percentage of OAE relative to the total workload ranged from $0.5-29 \% .^{1}$ If all cases where echocardiography might be indicated (table 1) were referred from the community, there would potentially be an unmanageable flood.

Although substantial cost savings may ensue in the long term, ${ }^{7}$ additional funding is required to initiate and run OAE services. Rarely, small schemes might be absorbed within existing hospital workloads, but supplying this type of service to a community of perhaps 200000 people is likely to be a major undertaking and require substantial additional funding.

Approximately one third of existing OAE services received funding from pharmaceutical companies. ${ }^{1}$ The obvious attraction of a mutually advantageous partnership between the private and public sectors needs to be treated with caution. There is clearly potential for ethical pitfalls where a test, essentially paid for by a particular pharmaceutical company, indicates the need for a drug produced by the same company. In our opinion, as elsewhere in medicine, commercial involvement by industry should not be discouraged but needs to be managed carefully.

Where the desire for OAE has been identified locally as a clinical priority, the service itself should be appropriately funded from NHS resources. In particular, knock on effects such as increased (as opposed to reduced) referrals for cardiological opinion need to be carefully considered.

\section{Conclusion}

At a time when there is increasing pressure to expand OAE provision, it is important to consider the potential weaknesses as well as the strengths of this type of service. It may well allow more patients to be managed effectively at low cost and with shorter waiting time than by standard cardiological referral, and is most suitable for requests with a well focused question amenable to a technical report. However, patients with more complex problems or evidence of significant symptomatic cardiac disease probably need to be referred to a cardiologist. In these circumstances, investment in the establishment of a rapid access, one stop cardiological assessment clinic would be desirable.

1 Rimington HM, Adam G, Chambers JB. Open access echocardiography. Lancet 1996;348:555-6.

2 Kerrigan DD, Brown SR, Hutchinson GH. Open access gastroscopy; too much to swallow. BMf 1990;300:374-6.

3 Francis CM, Carvana L, Kearney P, et al. Open access echocardiography in management of heart failure in the community. BMF 1995;310:634-6.

4 Wheeldon NM, MacDonald TM, Flucker CJ, et al. Echocardiography in chronic heart failure in the community. Quart $\mathscr{f}$ Med 1993;86:17-23.

5 McKee PA, Castelli WP, McNamara PM, et al. The natural history of congestive heart failure; the Framingham study. N Engl f Med 1971;285:1441-6.

6 Garg R, Yusef S. Overview of randomised trials of angiotensin-converting enzyme inhibitors on mortality and morbidity in patients with heart failure. $7 A M A$ 1995;273. 1450-6.

7 Scott WG, Scott HM. Heart failure-a decision analytic analysis of New Zealand data using the published results of analysis of New Zealand data using the published results of 156-67.

8 McMurray J, Hart W, Rhodes G. An evaluation of the cost of heart failure to the National Health Service in the UK. of heart failure to the National Healt

9 Walma EP, Hoes AW, vanDooren C, et al. Withdrawal of long term diuretic medication in elderly patients: a double blind randomised trial. BMF 1997;315:464-8.

10 Rodger JC, Shanks R, Hamill RA. Open access echocardiography: experience in a district hospital [abstract]. $\mathrm{Br} \mathrm{Heart} \mathcal{f}$ 1995;73 (suppl 3):14

11 McColl E, Newton J, Hutchinson A. An agenda for change in referral - consensus from general practice. $\mathrm{Br} \mathcal{7}$ Gen Pract 1994;44:157-62.

12 Ayenian JZ, Hauptmann PJ, Guagagnoli E, et al. Knowledge and practices of generalists and specialist physicians regarding drug therapy for acute myocardial infarction. $N$ Engl f Med 1994;331:1136-42.

13 Murphy JJ, Frain JPJ, Bossingham CM. "Open access" to echocardiography for suspected heart failure: does it work? [abstract] Br Heart f 1995;73 (suppl 3):39.

14 Mair FS, Bundred PE. The diagnosis and management of heart failure: GP opinions. Br f Cardiol 1996;3:121-5.

15 Wallace M, Levy H. Open access echocardiography. Study's conclusion is misleading and cannot be generalised [letter; comment]. BMF 1995;311:326-8.

16 MacFadyen RJ, MacDonald TM, Clarkson P, et al. Open access echocardiography. Single assessment may be dangerous. [letter; comment]. BMF 1995;311:327-8. 